\title{
High-field wave packets in semiconductor quantum wells: A real-space finite-difference time-domain formalism
}

\author{
S. Hughes* \\ Department of Physics, University of Surrey, Guildford GU2 7XH, United Kingdom
}

(Received 14 November 2003; published 13 May 2004)

\begin{abstract}
An untraditional space-time method for describing the dynamics of high-field electron-hole wave packets in semiconductor quantum wells is presented. A finite-difference time-domain technique is found to be computationally efficient and can incorporate Coulomb, static, terahertz, and magnetic fields to all orders, and thus can be applied to study many areas of high-field semiconductor physics. Several electro-optical and electromagneto-optical excitation schemes are studied, some well known and some new, and predicted observables are shown to be in qualitative agreement with experiment, where known.
\end{abstract}

DOI: $10.1103 /$ PhysRevB.69.205308

PACS number(s): 72.20.Ht, 42.50.Md, 71.35.-y, 78.47.+p

\section{INTRODUCTION AND MOTIVATION}

As the development of lasers continues to produce stronger and stronger fields, we can now study high-field-matter interactions in the laboratory, where the common theoretical techniques of nonlinear optics and perturbative field expansions break down. Indeed, it is now well established that the extremely-high-field physics of atomic ensembles presents many fascinating phenomena whereby the response of matter to high fields cannot be described within perturbation theory. For example, the process of high-harmonic generation (HHG) due to an intense atom-field interaction has received substantial attention in recent years, ${ }^{1}$ and harmonically generated coherent x-ray transients as short as 100 attoseconds have been predicted and indeed are beginning to be observed. ${ }^{2}$ The theoretical problem of HHG is most tactfully treated nonperturbatively by exploring the wave-packet (WP) motion by essentially exact numerical methods. For Rydberg atoms, higher-frequency harmonics are produced from continuum-state to bound-state transitions, in which electrons release the energy absorbed from the field during their journey in the continuum.

In certain limits there is a one-to-one mapping between the theoretical description of atoms and excitonsCoulombically bound electron-hole $(e-h)$ pairs in solids analogous to hydrogen. That said, the bound-state binding energies are substantially different. While the bound-state to continuum-state transitions in atoms are typically in the eV regime, in semiconductors these transitions are usually in the meV terahertz $(\mathrm{THz})$ regime. Nevertheless, with an increase in scientific research that utilizes free-electron lasers as well as $\mathrm{THz}$ solid-state emitters, high-field $\mathrm{THz}$ and mid-infrared (MIR) spectroscopy is now timely entering similar extreme regimes for semiconductors. ${ }^{3,4}$ However, the frequencies and field strengths (and thus ponderomotive energies) required are several orders of magnitude apart. For this reason, highfield effects in semiconductors can be observed at field intensities many orders of magnitude below what is required for the atoms.

The aim of this work is to employ a standard space-time method used frequently in electromagnetic simulation, and apply it to study semiconductor WP dynamics in the pres- ence of extreme fields-electric and (or) magnetic (dynamic and static). The advantages over previous techniques are many and will be discussed later. We will focus on $e-h$ WP's that are dynamically created by resonantly exciting a semiconductor with a short (but finite) optical pulse. The pulse intensity is weak and thus we work in the linear regime for the optical field, but all other fields will be treated nonperturbatively. This enables us to work in the low-density limit where many-body effects do not come into play. In the presence of large $\mathrm{THz}$, static, or (and) magnetic fields one can explore WP motion and experimental observables that are unique to the semiconductor environment. Moreover, semiconductor studies in the extreme-field regime, although relatively new, offer several advantages over atomic environments including the ability to change the effective masses and dimensionalities of the system.

At the outset, we emphasize that electro-absorption studies in semiconductors are certainly not new. The influence of a strong constant electric field $\mathbf{F}$ on the optical and electronic properties of semiconductors was introduced over 40 years ago, beginning with the Franz-Keldysh effect in bulk crystals. ${ }^{5}$ For two-dimensional (2D) semiconductors, such as quantum wells (QW's), ${ }^{6}$ similar effects occur for a field polarized in the plane, while the quantum-confined Stark effect occurs for fields polarized in the growth direction. In 1D, the Franz-Keldysh effect was only investigated recently. ${ }^{7}$

However, in the last few years there has been much interest in extending the semiconductor electro-optical studies into the dynamic, $\mathrm{THz}$, regime. Besides being of fundamental interest, as highlighted above there are some very close analogies with high-field effects in atoms, though this connection is rarely made in the literature. From a theoretical perspective, several approaches have been introduced, namely, Green function ${ }^{8}$ and nonequilibrium Green-function techniques $^{9}$ (both restricted in their handling of excitons and dynamics), as well as full scale numerical solution of the semiconductor Bloch equations (SBE) in momentum space. ${ }^{10}$ With regard to modeling high magnetic field interactions, usually one has to resort to a specialized basis set or (and) treat the Coulomb interaction perturbatively. In this work we set about solving the problem using a real-space and real time technique, specialized in the low-density regime (as in the above cases). The method (i) includes the 
fields ( $\mathrm{THz}$ and magnetic) and the Coulomb interactions nonperturbatively, (ii) is an order of magnitude faster than the SBE even when the latter excludes the magnetic field, and (iii) provides full spatial information thus providing a nice link with transport and optical properties. We will concentrate on QW's, where the technique can be immediately applied to recent free-electron laser studies and manipulation of excitonic states-recovering the dynamic Franz-Keldysh effect, ${ }^{3,10}$ the technique can, however, be applied to wells, dots, and bulk semiconductor materials. For the QW study, we also model magnetoexcitons and electro-magnetoexcitons and explore their WP motion. The present theoretical understanding of semiconductor optical processes in large magnetic fields is still fairly limited since including the Coulomb interaction is very difficult if one works in energy space; besides side stepping this problem, moreover, we show that in the presence of crossed magnetic and $\mathrm{THz}$ fields, WP stabilization can occur, just like in atomic physics experiments. Finally, we will explore extreme highfrequency induced sidebands that show good trends with recent MIR experiments, ${ }^{4}$ and give our conclusions.

\section{THEORETICAL AND COMPUTATIONAL METHOD}

High-field effects in atoms can be investigated nonperturbatively by numerically solving an effective Schödinger equation (in excitonic units):

$$
i \frac{\partial \Psi(\mathbf{r}, t)}{\partial t}=\left[-\nabla_{r}^{2}-V(\mathbf{r})+\mathbf{F}(t) \cdot \mathbf{r}\right] \mathbf{\Psi}(\mathbf{r}, t),
$$

where $\mathbf{r}$ is the spatial position, $\mathbf{\Psi}(\mathbf{r}, t)$ is the WP, $\mathbf{F}(t)$ is the oscillating electric field, and $V$ is the Coulomb potential. On the other hand, semiconductor optical problems are usually tackled within the framework of the SBE. ${ }^{11,12}$ In the lowdensity regime, this leads to the following set of equations for the polarization only:

$$
\frac{\partial \mathbf{P}_{\mathbf{k}}(t)}{\partial t}=-\mathbf{F}_{\mathrm{THz}}(t) \cdot \nabla_{\mathbf{k}}^{2} \mathbf{P}_{\mathbf{k}}-i \Delta_{\mathbf{k}} \mathbf{P}_{\mathbf{k}}(t)+i \mathbf{\Omega}_{\mathbf{k}}-\Gamma \mathbf{P}_{\mathbf{k}}
$$

where $\Delta_{\mathbf{k}}=E_{\mathbf{k}}-\omega_{l}+E_{g}, E_{g}$ is the band gap, $\omega_{l}$ is the carrier frequency of the optical pulse, and $\mathbf{F}_{\mathrm{THz}}$ is the applied $\mathrm{THz}$ field that can be polarized in any direction (see below). The generalized Rabi frequency is $\boldsymbol{\Omega}_{\mathbf{k}}=d_{c v} \tilde{\varepsilon}_{\mathrm{Opt}}+\Sigma_{\mathbf{q}} V_{\mathbf{k}-\mathbf{q}} \mathbf{P}_{\mathbf{q}}$, where $\widetilde{\varepsilon}_{\mathrm{Opt}}(t)$ is the slowly varying optical field polarized also in the QW plane, $V_{\mathbf{q}}$ is the Coulomb potential in momentum (i.e., k) space, and the total dephasing rate of the optical polarization $\Gamma=500 \mathrm{fs}^{-1}$ throughout; this is expected to be valid provided the input optical pulse is weak, which it is for this study. The solution of the SBE can then be solved in $\mathbf{k}$ space, and the total optical polarization is $\mathbf{P}_{\mathrm{Opt}}(t)$ $=2 \Sigma_{\mathbf{k}} d_{c v} \mathbf{P}_{\mathbf{k}}(t)$, where $d_{c v}$ is the interband dipole moment and the factor of 2 account for spin. However, we do not learn anything about the spatial dynamics, namely, $\mathbf{P}(\mathbf{r}$ $\neq 0, t)$; additionally, the $\mathbf{k}$ equations are extremely difficult to solve with the $\mathrm{THz}$ field since the components become anisotropic requiring a major computational effort. Indeed, the solution with the $\mathrm{THz}$ field alone was reported only fairly recently, ${ }^{10}$ and takes many days to simulate even with powerful computers. With the possible addition of a magnetic field, the numerical problem becomes intractable, requiring a switch to a large number of Landau basis states with some perturbation approximations. ${ }^{11}$ Thus a real-space approach is certainly more natural. We further highlight that the pioneering work of Schmitt-Rink and co-workers on the semiconductor Stark effect also approached the problem from the real-space perspective, essentially solving the stationary Schrödinger equation. ${ }^{6}$

Assuming on-resonance excitation (zero detuning) we can rewrite Eq. (2) in real space

$$
\begin{aligned}
i \frac{\partial \mathbf{P}(\mathbf{r}, t)}{\partial t}= & {\left[-\nabla_{r}^{2}+\mathbf{r} \cdot \mathbf{F}_{\mathrm{THz}}(t)-i \Gamma\right] \mathbf{P}(\mathbf{r}, t)-V(\mathbf{r}) \mathbf{P}(\mathbf{r}, t) } \\
& +\Omega(t) \delta(\mathbf{r}),
\end{aligned}
$$

where $\mathbf{P}(\mathbf{r}, t)$ is the $e$ - $h \mathrm{WP}$, and $\mathbf{r}=\mathbf{r}_{\mathbf{e}}-\mathbf{r}_{\mathbf{h}}$. A displacement of the WP from $\mathbf{r}=0$ means it is polarized, and thus the $\mathrm{THz}$ field will create an oscillating dipole. The optical polarization is now $\mathbf{P}_{\mathrm{Opt}}(t)=2 d_{c v} \mathbf{P}(\mathbf{r}=0, t)$. We have also killed two birds with the one stone since the WP approach gives us simultaneous optical and $\mathrm{THz}$ information. The THz-induced intraband dipole moment $\mathbf{P}_{\mathrm{THz}}(t)=e \int d r \mathbf{P}^{*}(\mathbf{r}, t) \mathbf{r} \mathbf{P}(\mathbf{r}, t)$, and for the emitted $\mathrm{THz}$ electric field-assuming a point source- $-\mathbf{E}_{\mathrm{THz}}(t) \propto \ddot{\mathbf{P}}_{\mathrm{THz}}(t)$. The structure of the above equation is very similar to the atomic problem but we have dephasing and an optical pulse; therefore the $e-h$ WP is created dynamically and relaxes dynamically (dephases). In the presence of a magnetic field $\mathbf{B}$ terms such as the momentum operator $\left(p_{x}^{2}=[i \partial / \partial x]^{2}\right)$ are replaced by $\left(p_{x}+e A_{x} / c\right)^{2}$ (Ref. 11) and do not pose any further refinements on the computational technique, which will be discussed in more detail below; relativistic correction terms can also be added. Thus, whether one studies free carriers, excitons, $\mathrm{THz}$ field ionization, or magnetic-field effects, the computational technique is the same. This is in stark contrast to the $\mathbf{k}$-space approach where the numerics become increasingly more difficult with the various fields. We do point out that the $\mathbf{k}$ space is, however, much better suited to high density and nonlinear optical studies, but intractable with large electric and magnetic fields.

Our numerical strategy is based on an exploitation of the finite-difference time-domain (FDTD) ${ }^{13,14}$ method; in the regime of complex electromagnetic scattering problems, FDTD has recently became the state-of-the-art computational method for solving Maxwell's equations almost exactly. Essentially we replace the polarization equation by finite-difference approximations, and implement them on a computational cell such as the Yee cell ${ }^{13}$ used in electrodynamics; an example of doing this for the 1D atomic problem (Schrödinger equation) is given in Ref. 14. For our present purpose, things are a little more complicated. To handle the fs optical-field source, we approximate the Dirac $\delta$ function by narrow normalized Gaussian and the numerical accuracy is verified to be in excellent agreement with the k-space approach in obtaining the quantitative excitonic and continuum 
properties of the quantum well, as will be shown below.

By way of an example, let us first label the real and imaginary parts of the WP by $\mathbf{P}^{R}$ and $\mathbf{P}^{I}$, respectively, where $\mathbf{P}(\mathbf{r}, t)=\mathbf{P}^{R}(\mathbf{r}, t)+i \mathbf{P}^{I}(\mathbf{r}, t)$. We will also drop the vector no- tation on the WP $(\mathbf{P}=P)$ and assuming an $x$-polarized $\mathrm{THz}$ field. Labeling the time t, and $x$ and $y$ spatial steps by $n, l$, and $m$, we can write out the WP FDTD difference equations corresponding to Eq. (3) as

$$
\begin{aligned}
\frac{P_{(n, l, m)}^{R}-P_{(n-1, l, m)}^{R}=}{\Delta t} & -\frac{\omega_{x}\left[P_{(n-0.5, l+1, m)}^{I}-2 P_{(n-0.5, l, m)}^{I}+P_{(n-0.5, l-1, m)}^{I}\right]}{[\Delta x]^{2}} \\
& -\frac{\omega_{x}\left[P_{(n-0.5, l, m+1)}^{I}-2 P_{(n-0.5, l, m)}^{I}+P_{(n-0.5, l, m-1)}^{I}\right]}{[\Delta y]^{2}} \\
& -x \cdot \mathbf{E}_{\mathrm{THz}}(t) P_{(n-0.5, l, m)}^{I}+\frac{\omega_{x}}{2\left(|\mathbf{r}|+a_{s}\right)} P_{(n-0.5, l, m)}^{I}-P_{(n-1, l, m)}^{R} \Gamma,
\end{aligned}
$$

and

$$
\begin{aligned}
\frac{P_{(n+0.5, l, m)}^{I}-P_{(n-0.5, l, m)}^{I}=}{\Delta t}= & \frac{\omega_{x}\left[P_{(n, l+1, m)}^{R}-2 P_{(n, l, m)}^{R}+P_{(n, l-1, m)}^{R}\right]}{[\Delta x]^{2}}+\frac{\omega_{x}\left[P_{(n, l, m+1)}^{R}-2 P_{(n, l, m)}^{R}+P_{(n, l, m-1)}^{R}\right]}{[\Delta y]^{2}} \\
& +x \cdot \mathbf{E}_{\mathrm{THZ}}(t) P_{(n, l, m)}^{R}-\frac{\omega_{x}}{2\left(|\mathbf{r}|+a_{s}\right)} P_{(n, l, m)}^{R}-P_{(n-0.5, l, m)}^{I} \Gamma+\frac{\Omega(t)}{2 \pi a_{d}} \exp \left[-\frac{r}{a_{d}}\right],
\end{aligned}
$$

where all spatial units are given in terms of the Bohr radius, $a_{0}$, and $\omega_{x}$ is the $(1 s)$ lowest state exciton binding frequency. The factor of 0.5 appearing in the time and spatial meshing (i.e., $n-0.5, n-1$, etc.) is consistent with the $E$ and $H$ field being displaced by half a time step and half a grid cell for regular electromagnetic FDTD. ${ }^{13,14}$ The numerically chosen parameters for the $\delta$ function and Coulombic screening are given by $a_{d}$ and $a_{s}$, which are both taken to be $0.3 a_{0}$. These parameters are needed to stop numerical divergence, but we highlight two important points: (1) as long as $a_{d}$ is small enough, the resulting spectra to be investigated below remain the same, and (2) $a_{s}$ physically corresponds to having a finite thickness quantum well, and thus is chosen to yield similar values to experimental spectra. For example, by including a realistic value we obtain the fundamental exciton binding energy of the QW that is about 2-3 times the bulk value, which is much closer to experimental values than the 2D quantum limit (four times the bulk). Furthermore, little quantitative changes take place by adjusting these parameters slightly. With regard to what time step to choose, we use the formula $d t=S[\Delta x]^{2} / \omega_{x}$, with $\Delta x=\Delta y$, and $S$ is the scaling factor to ensure numerical convergence. For all the tests below $S$ was set to 10 , though it can certainly be much smaller than this if less intense fields are applied, and we choose a total number of $x$ and $y$ points equal to 241 , on a regular spatial grid than span the range $\pm 36 a_{0}$. Finally, we note that it trivial and straightforward to add additional fields in the above FDTD equations, such as a magnetic field or other static-time-dependent fields. This is a clear advantage over fast Fourier transform split-step real-space methods commonly employed in high-field atomic optics. ${ }^{1}$

To this end, we work in the symmetric Gauge when mag- netic fields are applied, and focus attention here to a twosubband semiconductor QW excited by a short 50 fs optical pulse where the center of the pulse corresponds to time $t$ $=0$ fs. We take material parameters typical of $\mathrm{InGaAs} / \mathrm{GaAs}$ with a bulk exciton binding energy $E_{0}=4.2 \mathrm{meV}$, and Bohr radius $a_{0}=14 \mathrm{~nm}$.

\section{SIMULATION OF HIGH-FIELD WAVE PACKETS IN QUANTUM WELLS}

Initially we investigate four separate semiconductor excitations: (a) A short optical pulse only. (b) As in (a) but with the addition of a static magnetic field $\mathbf{B}=B_{0} \hat{\mathbf{n}}_{z}$ with a corresponding Landau frequency of $4 \mathrm{meV}$ (Ref. 11); $\hat{\mathbf{n}}_{z}$ is a unit vector in the perpendicular to the $\mathrm{QW}$ plane (growth direction). (c) As in (a) but with the addition of a $\mathrm{THz}$ electric field $\mathbf{F}_{\mathrm{THz}}(t)=\hat{\mathbf{n}}_{x} F_{0} \sin (\Omega t+\phi)$ with $\Omega=4 \mathrm{meV}$ and phase $\phi$ (at the center of the optical pulse $t=0$ ), $\hat{\mathbf{n}}_{x}$ a unit vector in the QW axis, $F_{0}$ the magnitude of the THz field is taken to be $5 \mathrm{kV} / \mathrm{cm}$; note that $4 \mathrm{meV}$ corresponds approximately to 1 THz. And (d), as in (a) but with a mixed $\mathbf{B}$ THz applied with identical parameters as above.

In Fig. 1 we show a snap shot of the corresponding WP's at $t=1.8 \mathrm{ps}$ after the short pulse has gone. $|\mathbf{P}(\mathbf{r}, t)|$ is a measure of the probability of finding electron and hole at position $\mathbf{r}$ at time $t$. Initially the WP is created at $\mathbf{r}=0$ to satisfy energy and momentum requirements of a direct gap semiconductor; note that much larger spatial regions are accounted for computationally. In (a) the WP is concentrated near the center due to the Coulomb interaction, since there is a high probability of finding the electron and hole at the same rela- 

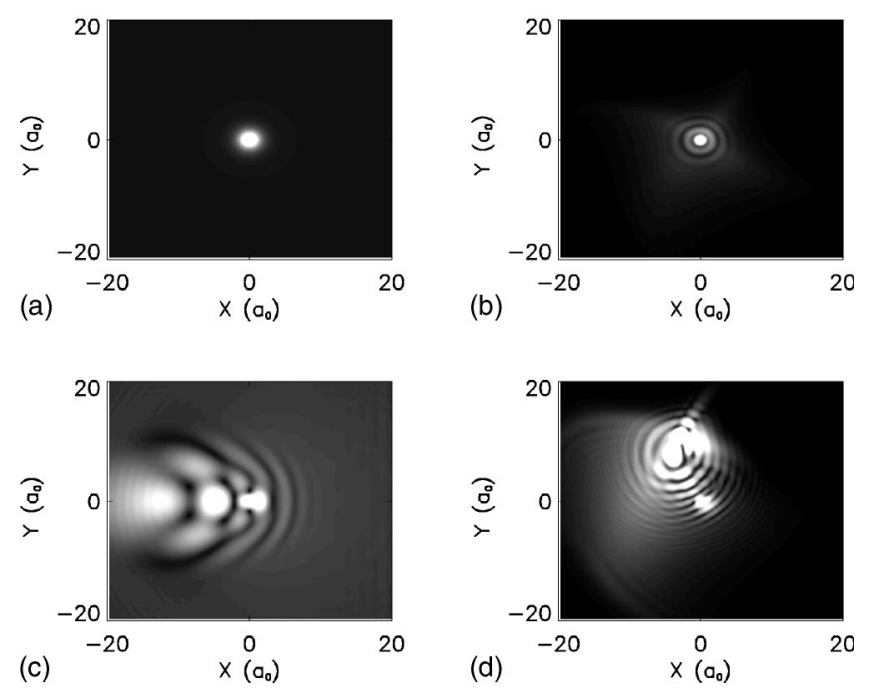

FIG. 1. Wave packet, $|\mathbf{P}(\mathbf{r}, t)|$, at time $t=1.8 \mathrm{ps}$ for various excitation regimes (a) optical pulse only, (b) also with a $\mathbf{B}$ field, (c) also with a THz field, and (d) also with a mixed B-THz field. The spatial dimensions are given in units of the exciton Bohr radius.

tive position (excitons). (b) Highlights the WP undergoing circular motion about the growth directions and small Landau structures begin to appear at larger spatial positions. (c) Depicts a pronounced WP distortion and interference because of broken symmetry and side lobes can be seen in the WP (these are formed by the combination of slow transverse spreading, the relatively fast field driven motion in the polarization direction, and Coulombic rescattering). (d) Shows a unique spiraling interfering WP due to the combined $\mathrm{THz}$ and $\mathbf{B}$ fields.

To link the WP motion to familiar optical properties, we simply Fourier transform the WP at $\mathbf{r}=0$ to the frequency domain and divide its imaginary contribution by the input optical pulse (via the Maxwell equations). In Fig. 2(a) we obtain the familiar field-free absorption spectrum for a QW, namely, a strong $1 s$ exciton resonance and a Coulombically enhanced continuum; in the presence of a $\mathrm{THz}$ field we obtain the dynamic Franz-Keldysh effect with oscillations above the band gap. ${ }^{3}$ Note that we have calculated the phase averaged WP results and thus the graphs represent the true absorption. Different phases, in principle, can coherently control the WP's motion, and phase effects are fully incorporated within our formalism. In Fig. 2(b) we obtain Landau oscillations and an enhancement of the lowest lying exciton. For clarity, ( $\mathbf{B}$ induced) diamagnetic shift terms of the band gap are not shown, though will depend on the helicity (spin dependent) of the optical light pulse, since $E_{ \pm}^{g}=E_{0}^{g} \mp \mu_{0}$ where $\mu_{0}$ is the Bohr magnetron. To further highlight our technique with larger fields, we also employ a $12 \mathrm{meV}$ Landau frequency, polarized, as before, in the growth direction. Substantial magnetoexcitons are seen in the inset, with peaks separated by the Landau frequency. These recover the known solution that employs many basis states in energy space, ${ }^{11}$ but without restrictions of perturbation theory, working with complicated basis states, and continuous wave $(\mathrm{CW})$ excitation. We also obtain the mixed $\mathbf{B}$-THz-field scenario resulting in interference between magnetoexcitons (Landau levels)
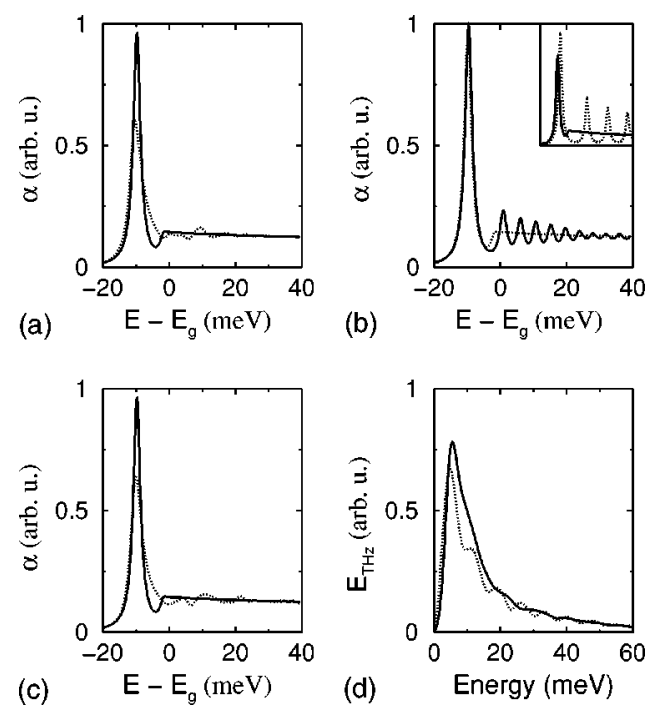

FIG. 2. Optical polarization vs energy corresponding to the following: (a) with (solid line) and without a THz field; (b) with (solid line) and without a B field; (c) with (solid line) and without a mixed B-THz field; (d) Emitted dipole field with a THz driving field only and a mixed B-THz field (solid line). In the inset we show the absorption spectrum, with (solid line) and without the $12 \mathrm{meV}$ (Landau frequency) B field.

and nonlinear $\mathrm{THz}$ interactions, shown in Fig. 2(c). In Fig. 2(d) we obtain the emitted $\mathrm{THz}$ field (from the oscillating dipole) in the presence of the $\mathrm{THz}$ driving field; a rich variety of harmonics akin to HHG in atoms appears. In the mixed field case we obtain an intriguing magnetic-THz coupling regime. The resolved peaks correspond to the absorption of one optical photon and the absorption (or emission) of one or more $\mathrm{THz}$ photons. Interestingly the harmonics are suppressed when we add a magnetic field since WP stabilization occurs for longer times; this effect is consistent with the Green-function prediction; ${ }^{15}$ all the physics can be explained from the WP's internal motion: the mixed-field WP is less distorted, and can be stabilized due to the forced Landau orbits and subsequently reduces exciton ionization (i.e., the WP propagating away from $\mathbf{r}=0$ ).

Next, we remove the time dependence of the oscillating $\mathrm{THz}$ field, i.e., $\mathbf{F}=F_{0} \hat{\mathbf{n}}_{x}$, thus working in the regime of the Franz-Keldysh effect. However, in addition we add a magnetic field in the growth direction. In Fig. 3(a) we show an example of the WP at time $t=1 \mathrm{ps}$. In this case, there is no longer a forced oscillating dipole though the WP is undergoing B-field spiraling and $e-h$ ionization through the applied dc field. The corresponding absorption is shown in Fig. 3(b), demonstrating pronounced exciton ionization and mixed Franz-Keldysh magnetoexciton features. We note that the emitted THz field, shows no clear peaks beyond the dc signal which is due to the fact that the static electric field is dominating the WP motion.

Finally, we present an example of extreme high-frequency $\mathrm{THz}$ optics by using a $5 \mathrm{THz}$ field with a $100 \mathrm{kV} / \mathrm{cm}$ amplitude. We have also tried such a simulation using a momentum space approach and it is intractable, yet the present method solved the problem in about $10-20 \mathrm{~min}$ on a stan- 


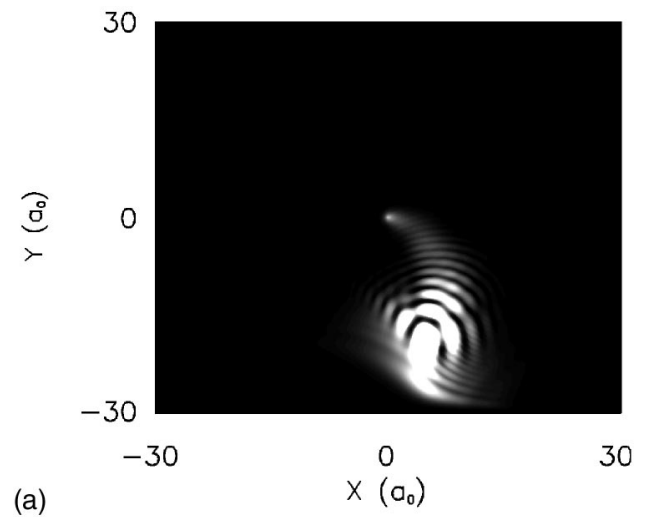

(a)

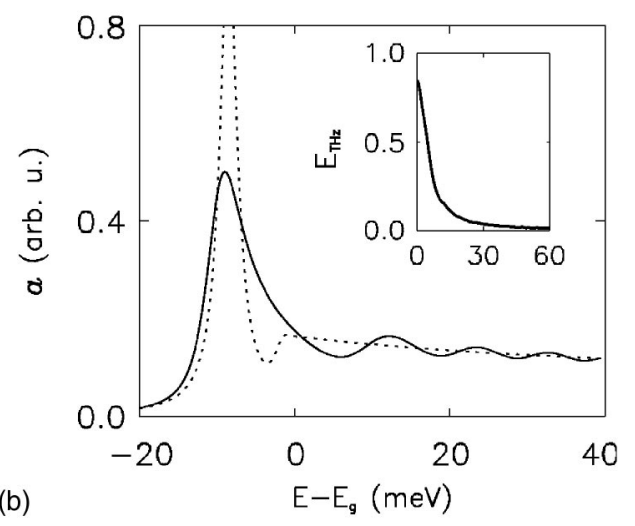

FIG. 3. (a) Wave packet at time $t=1$ ps for a static F-B field excitation. (b) Absorption spectrum with (solid line) and without the mixed field. As an inset we show the emitted dipole field.

dard computer workstation. In Fig. 4 we depict the WP at $t$ $=0.5 \mathrm{ps}$ as well as the optical absorption and $\mathrm{THz}$ emission. Changes in the absorption $100 \mathrm{meV}$ above and below the band gap can clearly be seen, and several broadband harmonics appear; both these effects show similar trends with recent experiments studying extreme MIR interactions in semiconductors. ${ }^{4}$ We remark that although the field is almost ten times the QW exciton bending energy, remarkably the Coulomb interaction still dominates the spatial interference patterns.

\section{CONCLUSIONS}

To conclude, we have presented a timely real-space-time method to calculate $e-h$ WP's in semiconductor quantum wells, allowing the theoretical study of nonperturbative field
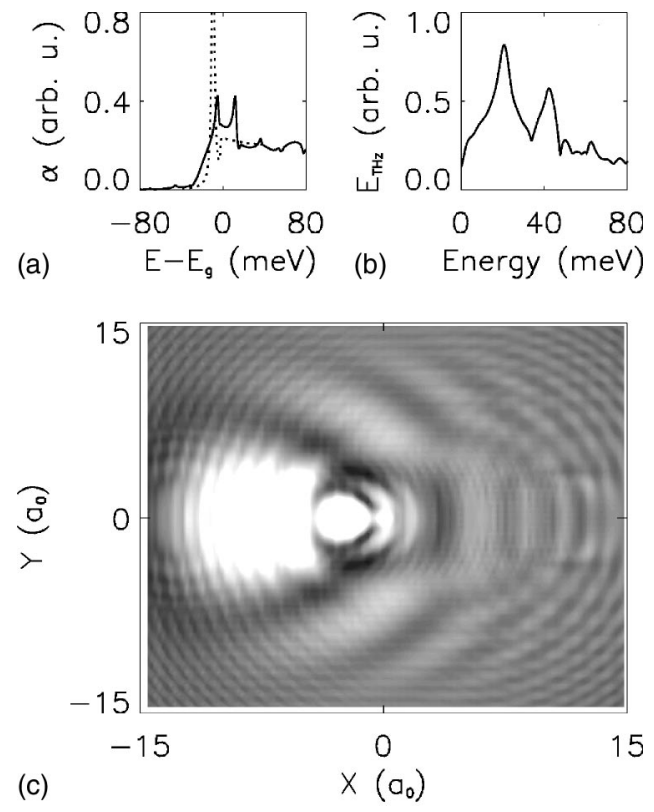

FIG. 4. (a) Absorption spectrum with (solid line) and without a large $100 \mathrm{kV} / \mathrm{cm}, 5 \mathrm{THz}$ field. (b) Emitted dipole field. (c) Wave packet at time $t=0.5 \mathrm{ps}$.

regimes in a very intuitive way. While the basic FDTD method is well known in the electromagnetic simulation field, it has not yet been applied to the complex problems that we address in this study, namely, high-field wave packets in semiconductors. The technique is shown to easily incorporate a variety of fields, such as Coulomb, magnetic, $\mathrm{THz}$, static - all included exactly, in the low-density limit. Furthermore, the technique is apparently much more efficient and powerful than other techniques such as $\mathbf{k}$-space SBE and nonequilibrium Green-function techniques, which only tackle a subset of what we have demonstrated here. Thus we anticipate that this method will, and should, be applied to study many areas of high-field physics, ranging from $\mathrm{HHG}$ in semiconductors and atoms to the coherent control of $e-h$ wave packets using magnets. Experimental observables are also easily extracted with the present FDTD method, and several examples for semiconductor quantum wells were shown to be in qualitative agreement with high-field experiments.

\section{ACKNOWLEDGMENT}

Discussions from D. S. Citrin and B. N. Murdin are gratefully acknowledged.

\footnotetext{
*Present address: NTT Basic Research Laboratories, NTT Corporation, 3-1 Morinosato-Wakamiya, Atsugi, Kanagawa 243-0198, Japan. Electronic address: hughes@ will.ntt.brl.co.jp

${ }^{1}$ See, for example, M. Protopapus, D.G. Lappas, and P.L. Knight, Phys. Rev. Lett. 79, 4550 (1997); G.G. Paulus, F. Zacher, H. Walther, A. Lohr, W. Becker, and M. Kleber, ibid. 80, 484 (1998); S. Kazamias, D. Douillet, F. Weihe, C. Valentin, A. Rousse, S. Sebban, G. Grillon, F. Aug, D. Hulin, and Ph. Balcou, ibid. 90, 193901 (2003); I.P. Christov, R. Bartels, H.C. Kapteyn,
}

and M.M. Murnane, ibid. 86, 5458 (2001); O.E. Alon, V. Averbukh, and N. Moiseyev, ibid. 85, 5218 (2000); M.W. Walser, C.H. Keitel, A. Scrinzi, and T. Brabec, ibid. 85, 5082 (2000).

${ }^{2}$ See, for example, P.B. Corkum, Nature (London) 403, 845 (2000); Y. Silberberg, ibid. 414, 494 (2001); J. Giles, ibid. 420, 737 (2002); M. Drescher, M. Hentschel, R. Kienberger, M. Uiberacker, V. Yakovlev, A. Scrinzi, Th. Westerwalbesloh, U. Kleineberg, U. Heinzmann, and F. Krausz, ibid. 419, 803 (2002); Hiromichi Niikura, F. Legare, R. Hasbani, M. Yu Ivanov, D.M. 
Villeneuve, and P.B. Corkum, ibid. 421, 826 (2003).

${ }^{3}$ K.B. Nordstrom, K. Johnsen, S.J. Allen, A.-P. Jauho, B. Birnir, J. Kono, T. Noda, H. Akiyama, and H. Sakaki, Phys. Rev. Lett. 81, 457 (1998); J. Kono, M.Y. Su, T. Inoshita, T. Noda, M.S. Sherwin, S.J. Allen, Jr., and H. Sakaki, ibid. 79, 1758 (1997).

${ }^{4}$ A.H. Chin, O.G. Calderon, and J. Kono, Phys. Rev. Lett. 86, 3292 (2001).

${ }^{5}$ W. Franz, Z. Naturforsch. Teil A 13, 484 (1958); L.V. Keldysh, Sov. Phys. JETP 34, 788 (1958).

${ }^{6}$ S. Schmitt-Rink, D.S. Chemla, and D.A.B. Miller, Adv. Phys. 38, 89 (1989).

${ }^{7}$ S. Hughes and D.S. Citrin, Phys. Rev. Lett. 84, 4228 (2000).

${ }^{8}$ D.S. Citrin and W. Hashawardhan, Phys. Rev. B 60, 1759 (1999).

${ }^{9}$ A.-P. Jauho and K. Johnsen, Phys. Rev. Lett. 76, 4576 (1996).

${ }^{10}$ S. Hughes and D.S. Citrin, Phys. Rev. B 59, R5288 (1999);
C.J. Dent, B.N. Murdin, and I. Galbraith, Phys. Rev. B 67, 165312 (2003).

${ }^{11}$ See H. Haug and S. W. Koch, Quantum Theory of the Optical and Electronic Properties of Semiconductors 3rd ed. (World Scientific, Singapore, 1994), and references therein.

${ }^{12} \mathrm{R}$. Zimmermann, Spectroscopy and Dynamics of Collective Excitations in Solids, edited by Di Bartoli, (Plenum, New York, 1997), p. 126.

${ }^{13}$ A Taflove, Computational Electrodynamics: The FiniteDifference Time-Domain Method (Artech House, Boston, London, 1995).

${ }^{14} \mathrm{D}$ Sullivan, Electromagnetic Simulation Using The FDTD Method, IEEE Press Series on RF and Microwave Technology (IEEE Press, New York, 2000).

${ }^{15}$ S. Hughes and D.S. Citrin, Solid State Commun. 113, 11 (2000). 